\title{
“MERCENARIOS, COMPAÑÍAS MILITARES Y DE SEGURIDAD PRIVADAS: DINÁMICAS Y RETOS PARA AMÉRICA LATINA",
}

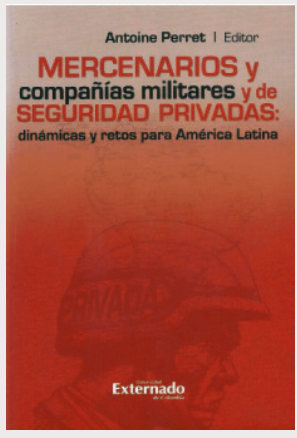

Título: "Mercenarios, compañías militares y de seguridad privadas: Dinámicas y retos para América Latina", Antoine Perret (Editor), 2010, Universidad Externado de Colombia

\section{Introducción}

Los colaboradores del libro editado por Antoine Perret provienen en su mayoría de la Red Investigativa Madison sobre las empresas privadas militares y de seguridad o Grupo Madison. Es una red de investigación que se formó a raíz del trabajo en conjunto de varios expertos, académicos y miembros del grupo de trabajo de las Naciones Unidas sobre el uso de mercenarios. La red se reunió por primera vez entre el 31 de enero y el 3 de febrero de 2008, en Madison, Wisconsin (USA) y de ahí obtuvo su nombre.

El grupo se auto declara como una "red de investigación independiente, que trabaja paralelamente con el mandato del grupo de trabajo de la ONU sobre el uso de mercenarios". El enfoque investigativo abarca las tendencias y temas asociados a la privatización de la seguridad. Se propone "dibujar las estructuras que acompañan los diversos niveles de la industria" y "compartir información, para evaluar y activar los estándares y estructuras de regulación existentes, con el fin de proponer nuevas estructuras y estándares que puedan regular y controlar la industria." Pone énfasis en los actores "más marginados" como lo son las poblaciones afectadas y los sub-contratistas de los países del sur.

\section{Síntesis}

El libro se divide en cuatro capítulos y una introducción que presenta el tema y las preguntas claves a tratar en los diferentes artículos. El Capítulo 1 se denomina: ¿Nuevo fenómeno, viejas ideas? y trata de las ambigüedades y dilemas de las Compañías Militares y de Seguridad Privada (CMSP). El Capítulo 2: América Latina: Escenario de desarrollo de las CMSP, trata de la creciente y reciente presencia de las CMSP en esta parte del mundo. El Capítulo 3 concretiza el fenómeno por medio de algunos estudios de caso. En el Capítulo 4 se presentan opciones de regulación de las CMSP. Los anexos adjuntan los protocolos correspondientes del derecho internacional de la ONU y da alguna información sobre los autores. 


\section{Introducción}

La introducción contesta algunas preguntas básicas sobre las CMSP dándole al lector la información necesaria para entender de lo que se trata. Especifica primero los servicios ofrecidos por ellos, como lo son: Protección armada de personas o de objetos, caravanas o edificios, mantenimiento y preparación de los armamentos, gestión de las cárceles, asesoramiento militar, inteligencia y entrenamiento de fuerzas locales y de personal de seguridad. Después explica que estos servicios se contratan mayoritariamente por los Estados, las Naciones Unidas y otras empresas privadas u Organizaciones No Gubernamentales y que la mayoría de las CMSP son de origen estadounidense o de Sudáfrica.

A continuación se describe la problemática tras el crecimiento de la industria de seguridad privada. Destacan sobre todo las siguientes preocupaciones: Existe un vacío jurídico y de regulación legal que se debe a la falta de definición y de estatus legal de las CMSP extranjeras en el ámbito nacional e internacional. Este vacío produce una falta de control por parte de las CMSP, especialmente para procesar judicialmente a los responsables de violaciones a los derechos humanos.

\section{Capítulo 1. ¿Nuevo fenómeno, viejas ideas?}

El primer capítulo retoma el problema del ambiguo estatuto del mercenario en el Derecho Internacional Humanitario (DIH) y ofrece una explicación al respecto. La regulación normativa internacional se encuentra en los Protocolos Adicionales I y II a los Convenios de Ginebra de 1949 que se hicieron durante la Conferencia de Ginebra en 1977. El autor resalta los siguientes puntos débiles del DIH al respecto:

1) El tópico del mercenarismo históricamente no había sido objeto de regulación por parte de los instrumentos o convenciones humanitarias internacionales y fue incluido solo en el curso de las discusiones de la conferencia diplomática de 1977. Hasta entonces el control del mercenariazgo era una responsabilidad y obligación de los estados neutrales.

2) La normativa del Protocolo Adicional I (PAI), artículo 47, solo diferencia al mercenario de la figura del combatiente y del prisionero de guerra y define qué se entiende por mercenario. La norma está "despojada de todo efecto concreto" porque su aplicación prácticamente depende de que alguien se declare mercenario para ser tratado como tal. Además, no distingue entre mercenario como persona individual y mercenarismo como fenómeno organizado (colectivos, empresas, personas jurídicas). Más que un efecto de regulación, esta norma legitimó y naturalizó al mercenario.

3) La poca diferencia entre la seguridad pública y la seguridad privada ha causado una confusión respecto al tipo de seguridad que el público puede esperar de los agentes privados de seguridad. Eso conlleva el riesgo de que los actores se aprovechen de esta confusión para legitimar el uso de la violencia por parte de actores no estatales si no se implementan mecanismos e instrumentos eficaces para responsabilizar en caso de crímenes cometidos por parte de mercenarios o agentes de seguridad privada.

\section{Capítulo 2. América Latina : Escenario de desarrollo de las CMSP}

El segundo capítulo presenta el enfoque regional del libro, introduciendo las diferentes modalidades de mercenarismo en América Latina y los problemas que se han generado alrededor de ellas. Los autores hacen hincapié en las siguientes tres: 
"Mercenarios, compañías militares y de seguridad privadas: Dinámicas y retos para América Latina"

1) Control de espacios geoestratégicos. Se refiere a la relación entre empresas de seguridad privada y compañías multinacionales de extracción de recursos naturales (minería, petróleo, etc.). El problema son las confrontaciones que se han dado entre guardias privados, grupos ambientalistas y movimientos sociales.

2) Control de bases militares. Se refiere a la reducción del personal militar en las bases militares estadounidenses para dar cabida a contratistas privados. Los problemas que se han detectado son un incremento en el tráfico de armas, tráfico y consumo de estupefacientes, tráfico de sexo con menores de edad.

3) Contratación de personal latinoamericano por parte de las CMSP. Se refiere a ex militares o ex policías contratados para trabajar como guardias de seguridad en Afganistán y otros conflictos armados. Los problemas consisten en que los agentes contratados se desvían de sus tareas originales como guardias, para ser usados en operativos de carácter militar.

Con base en estos hechos, se mencionan los siguientes potenciales efectos negativos de la industria de seguridad privada en los países latinoamericanos:

1) Una erosión de la justicia transnacional.

2) Una reducción en la capacidad de instituciones democráticas de regular actores armados.

3) Una reducción de la voluntad estatal de implementar regulaciones existentes.

4) Una apertura del territorio para operaciones militares privatizadas.

\section{Capítulo 3. Estudios de caso}

Los estudios de caso parten de una propuesta teórica que caracteriza a las CMSP como agencias de protección. En ella se mantiene que las CMSP surgen a raíz de situaciones dónde hay una lucha violenta por el poder local. Bajo esa condición se genera una demanda por mayor seguridad (un mercado abierto de protección) por la cual las CMSP compiten con sus servicios, como por ejemplo en Colombia.

También se presenta evidencia empírica sobre la insuficiencia de los marcos regulatorios en el ámbito nacional a partir del caso de Chile. En este país las CMSP se regulan por un conjunto de leyes e instancias. Adicional a la norma base sobre el "funcionamiento de los vigilantes privados" del año 1981 (Decreto de Ley No. 3607), influyen también la Ley de Control de Armas (No. 17.798) y el Código de Trabajo. Entre las instancias encargadas de supervisar y vigilar a las CMSP están los diferentes niveles de la Dirección General de Carabineros y el Ministerio del Interior y el de Defensa.

Por último, la perspectiva se eleva al ámbito internacional dónde se presenta el marco jurídico internacional enfocado en la responsabilidad de los Estados. Haciendo un desglose de las diferentes resoluciones de la Asamblea General de las Naciones Unidas, se evidencia que la responsabilidad de los Estados por la judicialización de las violaciones cometidas por las CMSP encuentra una justificación contundente en el DIH. 
Las resoluciones ratifican las siguientes responsabilidades:

Ningún funcionario podrá cometer, tolerar o justificar tratos o penas inhumanos (resolución 34/169 de 1979).

Revisar y adaptar periódicamente su legislación y prácticas vigentes para impedir actos que constituyan graves abusos de poder (resolución 40/34 de 1985).

Ponerle fin a las violaciones del DIH y ofrecer seguridades y garantías adecuadas de no repetición (resolución adoptada por la Asamblea General de las Naciones Unidas en 2002).

De la misma manera se encuentran llamados a la responsabilidad estatal en los principios y directrices de la Comisión de Derechos Humanos. Ahí los Estados son responsables de investigar, procesar, juzgar y condenar imparcialmente las violaciones a los derechos humanos que se den dentro de sus respectivos territorios. Así también, aplicar, respetar y asegurarse que se respeten las normas internacionales de derechos humanos y el derecho internacional humanitario. Y por último, cooperar y ayudar a los órganos judiciales internacionales competentes a investigar y perseguir penalmente las violaciones a los derechos humanos o al derecho internacional humanitario.

En América Latina ese rol le corresponde a la Corte Interamericana de Derechos Humanos, cuyo marco interpretativo ofrece más pautas para la elucidación de la responsabilidad estatal, en cuanto:

Impone a los Estados la obligación positiva de tomar medidas preventivas frente a riesgos que saben o deberían saber las autoridades correspondientes.

Existe incumplimiento de normas internacionales cuando un Estado no persigue actos de violaciones a los derechos humanos dentro de su territorio o cuando tolere que individuos o grupos cometan tales violaciones. En estos casos los Estados deben abstenerse de recurrir a figuras excluyentes de responsabilidad (e. g. amnistía) o a medidas que pretendan impedir la persecución o suprimir los efectos de una sentencia condenatoria.

\section{Capítulo 4. Opciones de regulación de las CMSP}

El hecho de que no existan regulaciones para las CMSP relega a los investigadores a buscar precedentes que podrían dar pautas para tales efectos. El primer artículo del último capítulo ejemplifica ese procedimiento a partir de la regulación de las Empresas Transnacionales (ETN) y encuentra precedentes en los Tribunales de Nuremberg dónde se juzgaron las violaciones cometidas por empresas alemanas durante la segunda guerra mundial. Así que sí existen instrumentos en el derecho internacional que señalan, aunque de manera imprecisa, los compromisos de las ETN en torno a los derechos humanos y su eventual responsabilidad frente a su incumplimiento.

Sin embargo, es frecuente encontrarse con una serie de problemas en el ámbito internacional cuando se trata de la implementación y sanción de estos instrumentos. Primero, hay una falta de consenso entre los Estados en torno a formular un instrumento con obligaciones claras, expresas y vinculantes para dichos actores (ETN). No todos los Estados ratifican las resoluciones de la ONU, y 
a menudo no hay posibilidades de hacerlos exigibles ante un tribunal internacional. Por último, la Corte Penal Internacional no consagra la posibilidad de juzgar a personas jurídicas.

Estas complicaciones se agravan en el ámbito nacional como la autora lo demuestra para el caso de Estados Unidos. The Alien Tort Claims Act confiere competencia a las cortes de distrito estadounidense para evaluar demandas interpuestas por extranjeros, siempre y cuando se trate de violaciones al derecho internacional o a un tratado de Estados Unidos. Pero para las ETN demandadas sólo se trata de responsabilidades civiles sin consecuencias internacionales y con requisitos y obstáculos "casi insuperables" para obtener una reparación.

El último artículo retoma estos problemas y propone una regulación de las CMSP en diferentes ámbitos con los siguientes componentes:

\section{Vigilancia internacional}

Una convención internacional para regular las actividades de las CMSP supervisada por un grupo de vigilancia internacional como parte de la ONU, permitiendo un seguimiento de las actividades de las CMSP por regiones o zonas. Ese grupo dispondría de un registro actualizado de todas las empresas que ofrecen servicios de seguridad no combativos. Los que incurren en combate serían retirados de la lista y quedarían fuera del mercado internacional. Esta lista pública podría ser complementada por una lista no pública que contenga los nombres de los empleados de las empresas para poder controlar mejor las actividades realizadas en cada zona. En caso de violación, las CMSP deberían presentarse a la justicia del país donde actúan y entregar a los responsables de los hechos, so pena de ser retirado de la lista si no lo hacen. Los Estados tienen que cerciorarse de ser capaces de judicializar a los empleados de las empresas de seguridad que contraten. Toda persona o ente que contrate una CMSP tiene que reportarlo con el Estado correspondiente.

\section{Control balanceado en el ámbito nacional}

a) Obligación de las CMSP de registrarse y obtener una licencia (como por ejemplo ya lo hacen en Estados Unidos y Sudáfrica).

b) Proceso de control y transparencia de todos los contratos de las CMSP frente al poder legislativo (hasta ahora la mayoría de estos contratos quedan a juicio del ejecutivo). Todo demandante de CMSP en el ámbito nacional, debería contar con la aprobación del ejecutivo y legislativo o pasar por algún tipo de inspección en el Congreso.

c) Estados observantes del grupo internacional de vigilancia con el fin de que acaten la lista de CMSP avalada internacionalmente.

d) Los contratos con CMSP deberían contemplar claramente las obligaciones, derechos, y mecanismos de solución de controversias o resolución de conflictos, coherentes con los estándares del derecho internacional.

Los contratos tienen que establecer con claridad

1) Los objetivos de la contratación,

2) Los procedimientos que se deben seguir, y 
3) El marco jurídico nacional e internacional que se debe cumplir, los instrumentos para resolver circunstancias imprevistas, denominación de quienes vigilan la ejecución y a quienes hay que presentar resultados.

\section{Crítica}

\section{A. Pesimismo académico}

Existe una perjudicial actitud negativa frente al fenómeno que se expresa en muchas valoraciones dentro de los artículos que son meramente subjetivas o a lo mejor derivadas de la fuerte impresión que dejó la revisión de los casos de violaciones a los derechos humanos que se han dado y probablemente se sigan dando alrededor y dentro de las CMSP.

¿Pero si contemplamos la figura del mercenario o del guardia de seguridad sin esta actitud negativa frente al fenómeno del cual forma parte? ¿Qué es lo que vemos?

La figura del mercenario no es parte de la cadena de comando del ejército. La relación entre él y la persona por la que trabaja es contractual. Es una relación horizontal. Dentro de la organización existe probablemente una cierta jerarquía por cuestiones de diferencias en conocimiento y experiencia. Pero no existen los mismos niveles de rango y sub ordenamiento que se conoce de la organización militar. En caso de desobediencia no hay sanciones como las de los ejércitos dónde ello puede significar la muerte.

Al final, los mercenarios están dónde están porque aceptaron ese tipo de trabajo. Si no quieren seguir pueden de la misma forma, dejar el trabajo según lo expuesto en los contratos. O simplemente irse y enfrentar el riesgo (probablemente bastante mínimo) de que haya una demanda civil por parte del empleador que a su turno tendrá en el peor de los casos consecuencias económicas. El mercenario no jura sobre la bandera de ningún país.

Lo mismo aplica para los guardias de seguridad privada. En contraste con un policía que jura vigilar por el cumplimiento de las leyes de un país o una jurisdicción, el guardia de seguridad privada no tiene ninguna obligación en este orden. Él decide cómo reaccionar frente a una situación según su experiencia y conciencia. Estrictamente hablando, no existen para un guardia privado infracciones como para un policía. Para el guardia solo existe su tarea o misión y los diferentes medios de los cuales dispone para lograr el objetivo.

¿Cómo elige entonces sus medios? Lo que estamos viendo entonces, son combatientes y guardias que al final responden únicamente a su propia conciencia y no a las leyes o a un juramento patriótico. Por supuesto tiene que cumplir la misión, pero cómo lo hace, es su propia decisión. También están sujetos a las leyes. Pero cuando trabajan en un país extranjero al suyo pueden evitar ser juzgados y condenados saliéndose del mismo. De esta forma la pregunta primordial no es si queremos seguridad privada o como la podemos regular. De hecho, ya la tenemos en todas partes y también la estamos regulando aunque insuficientemente. La pregunta sería: ¿Es una persona lo suficientemente madura (de su conciencia) para portar un arma y hacer lo correcto en una situación o no? Eso exige (más que a nuevas leyes o sistemas de gobernanza multi nivel), un vigoroso control psico-social en el reclutamiento de personal para las CMSP y el control de armas en general. 


\section{B. La figura de la ONU}

Los autores se refieren a la ONU como a un actor imparcial, olvidando que la ONU también es un actor político con intereses propios. Si la ONU quiere regular las actuaciones de los Estados es, en primera instancia, siempre una potencial intromisión en la soberanía de los mismos. Un Estado siempre tiene una razón de ser. La ONU solo la tiene en cuanto sirve a los Estados para mejorar sus relaciones particulares. No debería la ONU, que no responde a ningún control democrático por parte de los pueblos, imponer obligaciones a los Estados. La ONU es un instrumento, no es un gobierno.

\section{Para interés del lector}

El libro reúne mucha información bastante útil para alguien que quiera hacerse una idea sobre el tema. Sin embargo, esta información está escondida dentro de los artículos y cuesta llegar a ella. Cada artículo tiene una introducción que se vuelve al final redundante porque tratan de lo mismo: La privatización de la seguridad. Es un ejercicio tedioso el leer cada vez sobre las diferentes aproximaciones de cada autor al tema ya que uno espera encontrar el marco regulatorio de las CMSP y no las predilecciones de los expertos sobre teorías y modos de pensar acerca de ello. La única excepción es el artículo de Yadira Castillo Meneses sobre las Empresas Transnacionales que va directo al grano.

Dr. Benjamín Badura

Universidad de Potsdam, Alemania 